\title{
COLOR IMAGE RETRIEVAL BASED ON FULL RANGE AUTOREGRESSIVE MODEL WITH LOW-LEVEL FEATURES
}

\author{
A. Annamalai Giri ${ }^{1}$ and K. Seetharaman ${ }^{2}$ \\ ${ }^{1}$ Department of Computer Science, Sri Kuvempu First Grade College , Kengal, \\ Channapatna, Ramanagara Dist, Bangalore, INDIA, \\ girikumar246@gmail.com \\ ${ }^{2}$ Department of Computer Science \& Engineering, \\ Annamalai University, Annamalai Nagar, INDIA, \\ kseethaddeau@gmail.com
}

\begin{abstract}
This paper proposes a novel method, based on Full Range Autoregressive (FRAR) model with Bayesian approach for color image retrieval. The color image is segmented into various regions according to its structure and nature. The segmented image is modeled to RGB color space. On each region, the model parameters are computed. The model parameters are formed as a feature vector of the image. The Hotlling $T^{2}$ Statistic distance is applied to measure the distance between the query and target images. Moreover, the obtained results are compared to that of the existing methods, which reveals that the proposed method outperforms the existing methods.
\end{abstract}

\section{KEYWORDS}

FRAR model, query image, target image, feature vector, spatial features.

\section{INTRODUCTION}

In computer vision, feature representation schemes are classified as low-level, intermediate, and high-level. The low-level features are represented at pixel level while the high-level features are represented with abstract concepts, and the intermediate-level features represent something in between them. In this paper, it is believed that the low-level features make clear physical meanings and also related to high-level perceptual concepts. Because, the low-level features such as color, texture and spatial orientation of the pixels play vital role in color image formation. The spatial orientation of the pixels forms a shape or structure in an image. Next, the color features refine and enhance the shapes, and perceptually distinguish the regions from each other. Thus, most of the works use low-level features such as color, shape, texture and spatial orientation, and that are used for mine and retrieve the images. One of the most important issues in an image retrieval system is the feature extraction process, where the visual content of the images is mapped into a new space called as feature space. Mainly, the key issue to develop a successful retrieval system relies on identifying and choosing the right features that represent the images as strong as possible. Feature representation of the images may include color $[1,2,3,4,5,6,9]$, texture $([3,7,10,11,13]$ and shape $[1,6,7,8]$ information.

David C. Wyld et al. (Eds) : COSIT, DMIN, SIGL, CYBI, NMCT, AIAPP - 2014 
It is observed from the literature that the low-level features such as texture attributes -- fine texture, coarse texture, texture description, and spatial orientation of the texture play a significant role in image processing, viz. image classification, segmentation, edge and boundary detection, etc. $\mathrm{Wu}$ et al. [10] proposed a texture descriptor for browsing and similarity retrieval, and they have reported that the descriptor yield good results. The spatial orientation of the textures is not still used effectively in image retrieval domain. These texture features are more effective than other low-level features. This motivated us to develop the proposed method.

In this paper, a novel technique based on Full Range Autoregressive (FRAR) model is proposed, which characterizes the texture properties such as spatial orientation of the texture and untexture or structure (edge, boundaries) properties. It also gives unique representation to the characterized textures. The FRAR model coefficients $\mathrm{K}, \alpha, \theta$ and $\phi$ are considered as features, because $\alpha$ represents the strength of the linear dependency of the pixels on its neighboring pixels; the other parameters $\theta$ and $\phi$ are associated with the circular functions sine and cosine and their values ranging from 0 to $1 ; \mathrm{K}$ is a real valued function and it follows t-distribution. Since the parameter, $\alpha$, represents the strength of the dependency of the pixels, it extracts the textures attributes, i.e. fine, coarse etc. The FVs are compared region wise to that of the feature vectors of the target images in the image database. The distance measure Hotlling $T^{2}$ Square [14] distance is employed to measure the distance between the query and target images.

\section{PROPOSED MODEL FOR IMAGE RETRIEVAL}

Let $X(k, l)$ be a two-dimentional random variable that represents the intensity value of a pixel at location $(k, l)$ in an image. It is assumed that the pixel, $X$, may include noise and is considered independent and identical to a distributed Gaussian random variable with discrete time space and continuous state space with mean zero and variance $\sigma^{2}$ and is denoted as $\varepsilon(k, l)$, i.e. $\varepsilon(\mathrm{k}, \mathrm{l}) \sim \mathrm{N}\left(0, \sigma^{2}\right)$. Thus, the images are assumed to be affected by a Gaussian random process.

Thus, we propose an FRAR model as in equation (1),

$$
\begin{gathered}
X(k, l)=\sum_{\substack{p=-M \\
p=q \neq 0}}^{M} \sum_{\substack{q=-M \\
p \neq}}^{M} X(k+p, l+q)+\varepsilon(k, l) \\
\text { where, } \Gamma_{\mathrm{r}}=\frac{\mathrm{K} \sin (\mathrm{r} \theta) \cos (\mathrm{r} \phi)}{\alpha^{\mathrm{r}}}
\end{gathered}
$$

and $\mathrm{K}, \alpha, \theta$ and $\phi$ are real parameters. The $\Gamma_{\mathrm{r}} \mathrm{s}$ are the model coefficients, which are computed by substituting the model parameters $\mathrm{K}, \alpha, \theta$ and $\phi$ in equation (2). The model parameters are interrelated.

The initial assumptions on the parameters are $\mathrm{K} \in \mathrm{R}, \alpha>1$, and $\theta, \phi \in[0,2 \pi]$. Further restriction on the range of the parameters is placed by examining the identifiability of the model. It is interesting to note that some of the models used in the previous works such as white noise, Markov random field models and autoregressive models with finite and infinite orders can be regarded as a special case of the proposed FRAR model.

Thus,

i ) if we set $\theta=0$, then the FRAR model reduces to the white noise process. 
ii ) when $\alpha$ is large, the coefficients $\Gamma_{r} s$ become negligible as $r$ increases. Hence, the FRAR model reduces to a $\mathrm{AR}(r)$ model approximately, for a suitable value of $r$, where $r$ is the order of the model.

iii ) when $\alpha$ is chosen to be less than one, the FRAR model becomes an explosive infinite order model.

The fact that $X(k, l)$ has regression on its neighborhood pixels gives rise to the terminology of Markov process. However, in this case, the dependence of $X(k, l)$ on neighborhood values may be true to some extent. In fact, the process is Gaussian under the assumption that the $\varepsilon(k, l) \mathrm{s}$ are Gaussian, and in this case, its probabilistic structure is completely determined by its second order properties. Second order properties meant for the proposed FRAR model is asymptotically stationary up to order two, provided $1-\alpha<\mathrm{K}<\alpha-1$. Finally, the range of the parameters of the model is set with the constraints $\mathrm{K} \in \mathrm{R}, \alpha>1,0<\theta<\pi, 0<\phi<\pi / 2$.

\section{PARAMETER ESTIMATION}

In order to implement the proposed FRAR model, we have to estimate the parameters. The parameters, $\mathrm{K}, \alpha, \theta$ and $\phi$ are estimated by taking the suitable prior information for the hyper parameters $\beta, \gamma$, and $\delta$, based on Bayesian methodology. Only for the computational purpose, the pixel values of each subimage are arranged as one-dimensional vectors $X(\mathrm{t}), \mathrm{t}=1,2,3, \ldots, \mathrm{N}$. Since, the error term $\varepsilon(k, l)$ in equation (1) is independent and identical to distributed Gaussian random variable, the joint probability density function of the stochastic process $\{X(\mathrm{t})\}$ is

$$
\mathrm{P}(\mathrm{X} / \Theta) \propto\left(\sigma^{2}\right)^{-\mathrm{N} / 2} \exp \left[-\frac{1}{2 \sigma^{2}} \sum_{\mathrm{t}=1}^{\mathrm{N}}\left\{\mathrm{X}_{\mathrm{t}}-\mathrm{K} \sum_{\mathrm{r}=1}^{\infty} \mathrm{S}_{\mathrm{r}} \mathrm{X}_{\mathrm{t}-\mathrm{r}}\right\}^{2}\right]
$$

where $X=(\mathrm{X} 1, \mathrm{X} 2, \ldots, \mathrm{XN}) ; \Theta=\left(\mathrm{K}, \alpha, \theta, \phi, \sigma^{2}\right)$ and $\mathrm{S}_{\mathrm{r}}=\frac{\sin (\mathrm{r} \theta) \cos (\mathrm{r} \phi)}{\alpha^{\mathrm{r}}}$.

When we analyse the real data with finite number of $\mathrm{N}$ observations, the range of the index $r$, viz. 1 to $\infty$, reduces to 1 to $\mathrm{N}$, and so the joint probability density function of the observations is given

in equation (3). The summation $\sum_{\mathrm{r}=1}^{\infty}$ can be replaced by $\underset{\mathrm{r}=1}{\mathrm{~N}}$ which gives

$$
\mathrm{P}(\mathrm{X} / \Theta) \propto\left(\sigma^{2}\right)^{-\mathrm{N} / 2} \exp \left[-\frac{1}{2 \sigma^{2}} \sum_{\mathrm{t}=1}^{\mathrm{N}}\left\{\mathrm{X}_{\mathrm{t}}-\mathrm{K} \sum_{\mathrm{r}=1}^{\mathrm{N}} \mathrm{S}_{\mathrm{r}} \mathrm{X}_{\mathrm{t}-\mathrm{r}}\right\}^{2}\right]
$$

By expanding the square in the exponent, we get

$$
\mathrm{P}(\mathrm{X} / \Theta) \propto\left(\sigma^{2}\right)^{-\mathrm{N} / 2} \exp \left[-\frac{1}{2 \sigma^{2}}\left\{\mathrm{~T}_{00}+\mathrm{K}^{2} \sum_{\mathrm{r}=1}^{\mathrm{N}} \mathrm{S}_{\mathrm{r}}^{2} \mathrm{~T}_{\mathrm{rr}}+2 \mathrm{~K}^{2} \underset{\substack{\mathrm{r}, \mathrm{s}=1 \\ \mathrm{r}<\mathrm{s}}}{\mathrm{N}} \mathrm{S}_{\mathrm{r}} \mathrm{S}_{\mathrm{S}} \mathrm{T}_{\mathrm{rs}}-2 \mathrm{~K} \underset{\mathrm{r}=1}{\stackrel{\mathrm{N}}{\mathrm{N}} \mathrm{S}_{\mathrm{r}} \mathrm{T}_{0 \mathrm{r}}}\right]\right]
$$




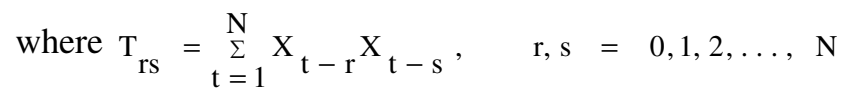

The above joint probability density function can be written as

$$
\mathrm{P}(\mathrm{X} / \Theta) \propto\left(\sigma^{2}\right)^{-\mathrm{N} / 2} \exp \left[-\frac{\mathrm{Q}}{2 \sigma^{2}}\right]
$$

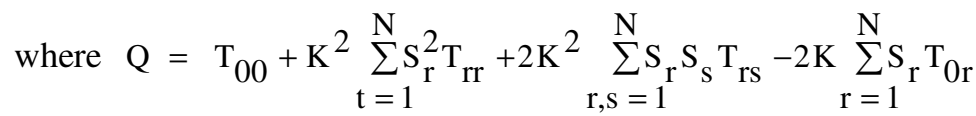

$$
\mathrm{r}<\mathrm{s}
$$

$\mathrm{K} \in \mathrm{R}, \alpha>1,0<\theta<\pi, 0<\phi<\pi / 2$ and $\sigma^{2}>0$.

So, the joint prior density function of $\Theta$ is given by

$$
\begin{array}{r}
\mathrm{P}(\Theta) \propto \beta \exp \left(-\beta(\alpha-1)-v / \sigma^{2}\right)\left(\sigma^{2}\right)^{-(\delta+1)} ; \\
\sigma^{2}>0, \alpha>1,0<\theta<\pi, 0<\phi<\pi / 2 .
\end{array}
$$

where, $\mathrm{P}$ is a general notation for the probability density function of the random variables given within the parentheses following $\mathrm{P}$.

Using (6), (8) and Bayes theorem, the joint posterior density of $\mathrm{K}, \alpha, \theta, \phi$ and $\sigma^{2}$ is obtained as

$$
\begin{gathered}
\mathrm{P}(\Theta / \mathrm{X}) \propto \exp (-\beta(\alpha-1)) \exp \left(-1 / 2 \sigma^{2}\right)(\mathrm{Q}+2 v)\left(\sigma^{2}\right)^{-\left(\frac{\mathrm{N}}{2}+\delta+1\right)} \\
\mathrm{K} \in \mathrm{R}, \alpha>1,0<\theta<\pi, 0<\phi<\pi / 2 \text { and } \sigma^{2}>0 .
\end{gathered}
$$

Integrating (9) with respect to $\sigma^{2}$, the posterior density of $K, \alpha, \theta$ and $\phi$ is obtained as

$$
\begin{gathered}
\mathrm{P}(\mathrm{K}, \alpha, \theta, \phi / \mathrm{X}) \propto \quad \exp (-\beta(\alpha-1))(\mathrm{Q}+2 \mathrm{v})^{-}\left(\frac{\mathrm{N}}{2}+\delta\right) \\
\mathrm{K} \in \mathrm{R}, \alpha>1,0<\theta<\pi, 0<\phi<\pi / 2
\end{gathered}
$$

where,

$$
[\mathrm{Q}+2 \mathrm{v}]=\left[\left(\mathrm{K}^{2} \sum_{\mathrm{r}=1}^{\mathrm{N}} \mathrm{S}_{\mathrm{r}}^{2} \mathrm{~T}_{\mathrm{rr}}+2 \mathrm{~K}^{2} \underset{\substack{\mathrm{r}, \mathrm{s}=1 \\ \mathrm{r}<\mathrm{s}}}{\mathrm{N}} \mathrm{S}_{\mathrm{r}} \mathrm{S}_{\mathrm{s}} \mathrm{T}_{\mathrm{rs}}-2 \mathrm{~K} \underset{\mathrm{r}=1}{\stackrel{\mathrm{N}}{\mathrm{N}} \mathrm{S}_{\mathrm{r}} \mathrm{T}_{0 \mathrm{r}}}\right)+\mathrm{T}_{00}+2 \mathrm{v}\right]
$$

That is,

$$
\begin{aligned}
(Q+2 v) & =a K^{2}-2 K b+T_{00}+2 v, \\
& =C\left[1+a_{1}\left(K-b_{1}\right)^{2}\right]
\end{aligned}
$$

where, 


$$
\begin{aligned}
& C=T_{00}-\frac{b^{2}}{a}+2 v \\
& a=\sum_{r=1}^{N} S_{r}^{2} T_{r r}+2 \sum_{\substack{r, s=1 \\
r<s}}^{N} S_{r} S_{s} T_{r s} \\
& \mathrm{~b}=\sum_{\mathrm{r}=1}^{\mathrm{N}} \mathrm{S}_{\mathrm{r}} \mathrm{T}_{0 \mathrm{r}} ; a_{1}=\frac{a}{C} ; b_{1}=\frac{b}{a}
\end{aligned}
$$

Thus, the above joint posterior density function of $\mathrm{K}, \alpha, \theta$ and $\phi$ can be rewritten as

$$
\begin{array}{r}
\mathrm{P}(\mathrm{K}, \alpha, \theta, \varphi / \mathrm{X}) \propto \exp (-\beta(\alpha-1))\left[\mathrm{C}\left\{1+\mathrm{a}_{1}\left(\mathrm{~K}-\mathrm{b}_{1}\right)^{2}\right\}\right]^{-\mathrm{d}} \\
\mathrm{K} \in \mathrm{R}, \alpha>1,0<\theta<\pi, 0<\varphi<\pi / 2
\end{array}
$$

where, $d=\frac{N}{2}+\delta$

This shows that given $\alpha, \theta$ and $\phi$ the conditional distribution of $\mathrm{K}$ is ' $\mathrm{t}$ ' distribution located at b1 with (2d-1) degrees of freedom.

The proper Bayesian inference on $\mathrm{K}, \alpha, \theta$ and $\phi$ can be obtained from their respective posterior densities. The joint posterior density of $\alpha, \theta$ and $\phi$, namely, $\mathrm{P}(\alpha, \theta, \phi / X)$, can be obtained by integrating (13) with respect to $\mathrm{K}$. Thus, the joint posterior density of $\alpha, \theta$ and $\phi$ is obtained as

$$
\begin{array}{r}
\mathrm{P}(\alpha, \theta, \varphi / \mathrm{X}) \propto \exp (-\beta(\alpha-1)) \mathrm{C}-\mathrm{d}_{\mathrm{a}_{1}}^{-1 / 2} \\
\quad \alpha>1, \quad 0<\theta<\pi, \quad 0<\phi<\pi / 2
\end{array}
$$

The point estimates of the parameters $\alpha, \theta$ and $\phi$ may be taken as the means of the respective marginal posterior distribution i.e. posterior means. With a view to minimize the computations, we first obtain the posterior mean of $\alpha$ numerically. Then fix $\alpha$ at its posterior mean and evaluate the conditional means of $\theta$ and $\phi$ fixing $\alpha$ at its mean. We fix $\alpha, \theta$ and $\phi$ at their posterior means respectively and then evaluate the conditional mean of $\mathrm{K}$.

Thus, the estimates are

$$
\begin{aligned}
\hat{\alpha} & =E(\alpha) \\
(\hat{\theta}, \hat{\phi}) & =E(\theta, \phi / \alpha=\hat{\alpha}) \quad \text { and } \\
\hat{\mathrm{K}} & =E(K / \hat{\alpha}, \theta=\hat{\theta}, \phi=\hat{\phi}) .
\end{aligned}
$$

The estimated parameters $\mathrm{K}, \alpha, \theta$ and $\phi$ are adopted to compute the coefficients $\Gamma_{\mathrm{r}} \mathrm{s}$ of the model in equation (1). The model parameters are utilised to compute the autocorrelation coefficient, which is used to identify the texture primitives and micro textures present in the image.

The parameters of the model are combined together and formed as FVs. The same kind of features are also extracted from the target image to be retrieved from the image database. These features are treated as two different FVs $\mathrm{x}$ and $\mathrm{y}$. 


\section{SIMILARITY MEASURE}

In order find the similarity of the query and target images, the Hotlling $T^{2}$ statistic $\left(\mathrm{T}^{2}\right)$ [14] expressed in equation (16) is applied on the feature vectors $\hat{\mathrm{x}}$ and $\hat{\mathrm{y}}$ of the query and target images.

$$
\mathrm{T}_{\mathrm{d}}^{2}(\mathrm{X}, \mathrm{Y})=\frac{\mathrm{n}_{\mathrm{x}} \cdot \mathrm{n}_{\mathrm{y}}}{\mathrm{n}_{\mathrm{x}}+\mathrm{n}_{\mathrm{y}}}(\overline{\mathrm{X}}-\overline{\mathrm{Y}})^{\prime} \mathrm{A}^{-1}(\overline{\mathrm{X}}-\overline{\mathrm{Y}})
$$

Where,

$$
\bar{X}=\frac{1}{n_{x}} \sum_{i=1}^{n_{x}} X_{i} \quad \bar{Y}=\frac{1}{n_{y}} \sum_{i=1}^{n_{y}} Y_{i}
$$

are the sample means, and

$$
\begin{aligned}
& A_{x}=\frac{\sum_{i=1}^{n_{x}}\left(X_{i}-\bar{X}\right)\left(X_{i}-\bar{X}\right)^{T}}{n_{x}-1} \\
& A_{y}=\frac{\sum_{i=1}^{n_{y}}\left(Y_{i}-\bar{Y}\right)\left(Y_{i}-\bar{Y}\right)^{T}}{n_{y}-1} \\
& A=\frac{A_{x}+A_{y}}{n_{x}+n_{y}-2}
\end{aligned}
$$

is the unbiased pooled covariance matrix of the query and target images.

If $\mathrm{T}_{\mathrm{d}}^{2} \leq \frac{\left(\mathrm{N}_{\mathrm{x}}+\mathrm{N}_{\mathrm{y}}-2\right) \mathrm{p}}{\mathrm{N}_{\mathrm{x}}+\mathrm{N}_{\mathrm{y}}-\mathrm{p}-1} \mathrm{~F}_{\alpha ; \mathrm{N}_{\mathrm{x}}+\mathrm{N}_{\mathrm{y}}-\mathrm{p}-1}$, then it is inferred that the query and target images are same or similar (i.e., belongs to the same class); otherwise, the two images are different (i.e. belongs to different classes). $\mathrm{F}_{\alpha ; \mathrm{N}_{\mathrm{x}}+\mathrm{N}_{\mathrm{y}}-\mathrm{p}-1}$ represents values in statistical F-table at the level of significance $\alpha$ with degrees of freedom $\mathrm{N}_{x}+\mathrm{N}_{-}-\mathrm{p}-1 ; \mathrm{p}$ represents the size of the feature vectors. Based on the distance values, the images are marked and indexed in ascending order, and the indexed images are retrieved.

\section{MEASURe OF PERformanCE}

In order to validate the performance of the proposed method, the precision and recall measures [12] are used, which are given in equations (17) and (18).

$$
\operatorname{Pr} \text { ecision }(\mathrm{P})=\frac{\mid\{\text { retrieved images }\} \text { I \{relevent images }\} \mid}{\mid \text { retrieved images } \mid}
$$




$$
\operatorname{Recall}(\mathrm{R})=\frac{\mid\{\text { retrieved images }\} \mathrm{I} \text { \{relevant images }\} \mid}{\mid \text { relevant Images } \mid}
$$

Where $|\cdot|$ returns the size of the set. The precision $(\mathrm{P})$ represents the ratio of the number of images relevant to the query image among retrieved images to the number of retrieved images. The recall $(\mathrm{R})$ represents the ratio of the number of images relevant to the query image among retrieved images to the number of images relevant to the query image.

\section{EXPERIMENTS AND RESULTS}

In order to implement the proposed method, 1277 color images of size $512 \times 512$ pixels have been collected from various sources such as 293 texture images from Brodatz Album; 488 images from Corel image database; 496 images from VisTex image database; and 268 images with size $128 \times 128$ are photographed by a digital camera; 257 images with size $128 \times 128$ have been downloaded from various websites. To examine the proposed system is invariant for rotation and scaling; the images are rotated through $90^{\circ}, 180^{\circ}$ and $270^{\circ}$ degrees, and scaled. Based on this image collection, an image database and their FV database are constructed.
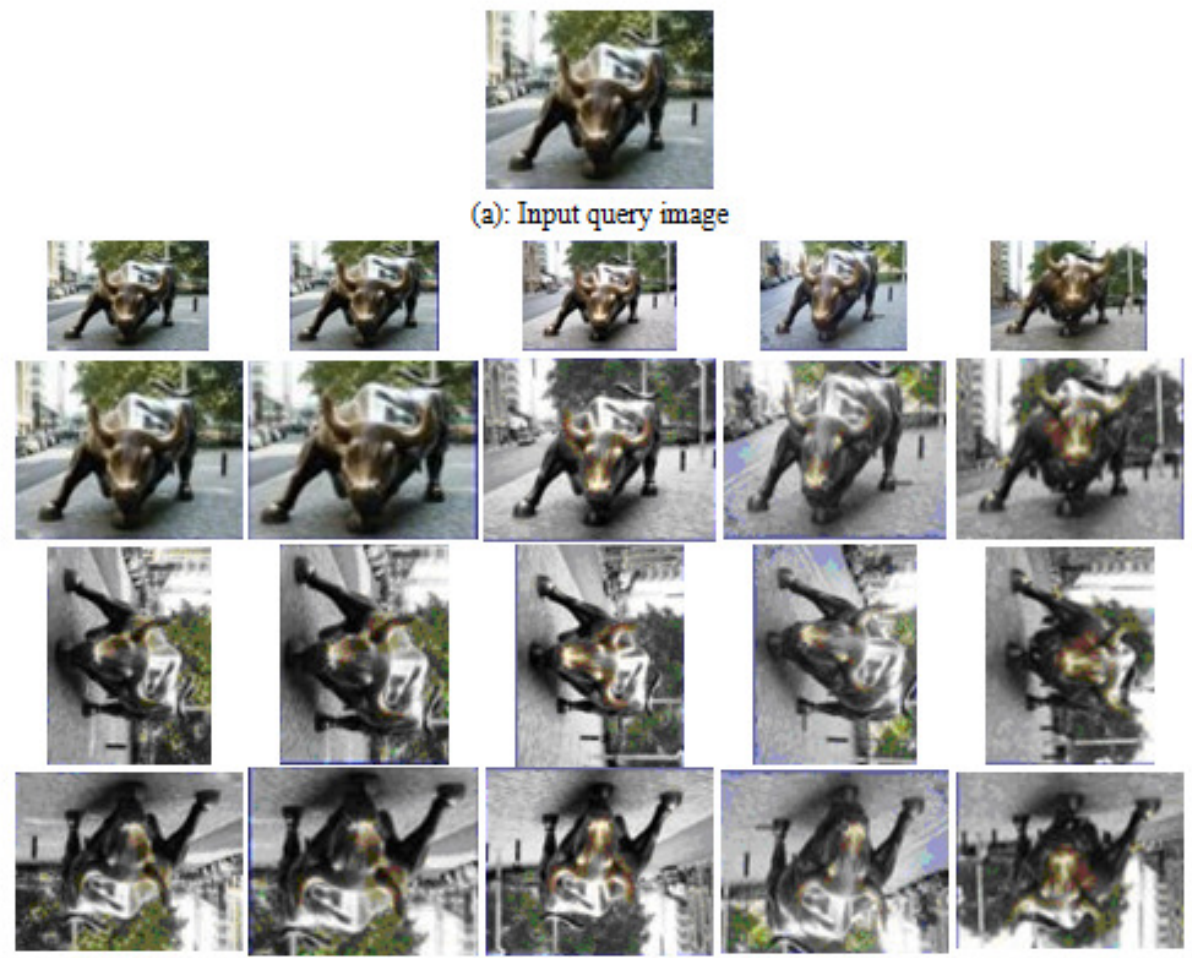

(b): Retrieved output images

Figure 1. Wall Street Bull - downloaded from internet. (a): Input query image; (b): Retrieved output images: row 1 - scaled down image of size $75 \times 100$; row 2 - actual image with size $96 \times 128$; row 3 images in row 2 are rotated clockwise by 90 degrees; row 4 - images in row 2 are rotated clockwise by 180 degrees.

The input query image is segmented into various regions according to its shapes and structure, and it is modelled to RGB colour space. The proposed method is employed on R, G and B components individually for extracting the image features as discussed in sections 2 and 3 . On each region, the parameters and autocorrelation coefficients are computed, and they are combined 
together and formed as FVs database. The extracted features are classified into various groups according to their nature using fuzzy c-means algorithm [13]. For each group, median value is calculated, and based on it the FVs are indexed. Based on the classes of the FVs, images in the database are classified into different groups, and it establishes a link between the images and the corresponding FVs of each class. Now, the extracted FVs of the query image are compared to that of the index of the FVs in the image feature database and is identified using the expression given in equation (15). Then the FVs of the query image are matched with the FV database, and retrieve the same or similar images from that class. If the FVs of the query image do not match with any classes of the FV database, then it is formed as a new FV class.

If the given input image is structured, it is segregated into various regions according to its shapes and structure; if it is texture image, then it considered as it is for retrieving the same or similar images from the image database. The input query image is identified whether it is texture or structure by computing the coefficient of variation $(\mathrm{CV})$, and the $\mathrm{CV}$ value is compared to a threshold value $t$. If $C V>t$ then it is assumed that the input image is structured image, and if CV $<t$ then the input image is assumed to be texture image. The threshold $t$ is fixed as $25 \%$.

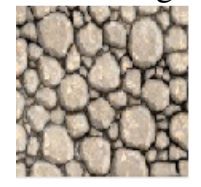

(a): Input query image
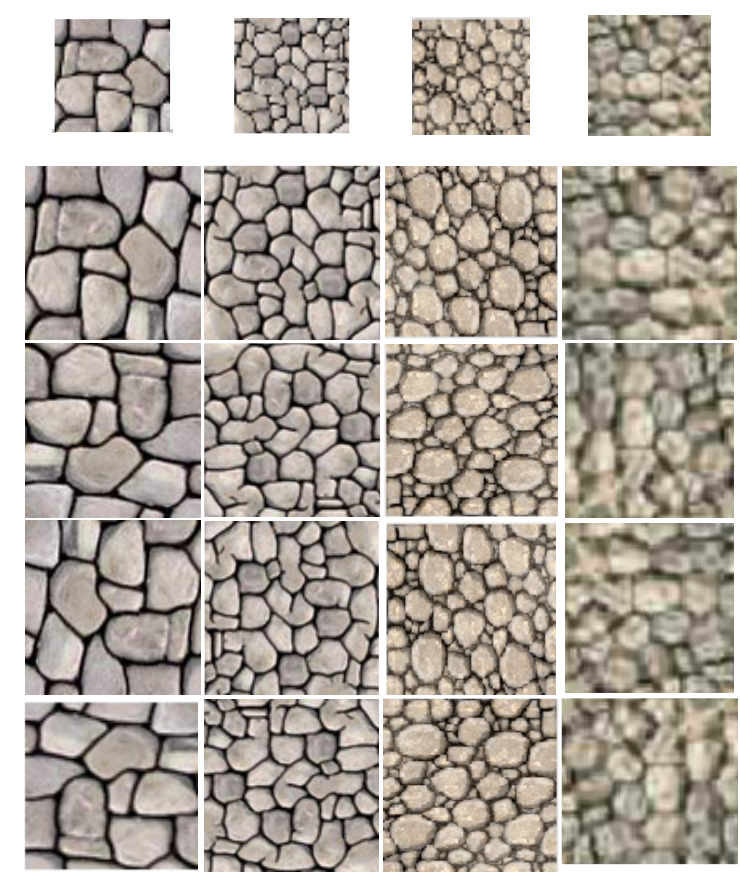

(b): Retrieved output images

Figure 2: VisTex image database - Structural Texture Images: 1(a) - Input query image; 1(b) - row 1: scaled down image; row 2: actual images; row 3: actual images rotated clockwise by 90 degree; row 4: actual images rotated clockwise by 180 degrees; row 5: actual images rotated clockwise by 270 degrees

In order to validate the proposed system, the image in Figure 1(a) is given as input query image to the system, and it retrieves the images in columns 1, 2 and 3 of Figure 1(b) while the level of significance is fixed at 0.001 ; the images in column $1,2,3$ and 4 are retrieved at 0.02 level of 
significance; at 0.01 significance level, the system retrieves the images in column 1, 2, 3, 4 and 7; at the level of significance 0.1, the system retrieves all the images presented in Figure 1(b).

To emphasis the effectiveness and efficiency of the proposed system, another type of structural texture image with stochastic pattern is given as input, which is presented in Figure 2(a). The proposed system retrieves the images in columns 3 of the Figure 2(b) when the level of significance is fixed at 0.001 ; the images in column 2,3 and 4 are retrieved at 0.04 level of significance; at 0.08 level of significance, the system retrieves all the images in Figure 2(b).

\section{DISCUSSION AND CONCLUSION}

In this paper, a novel scheme is proposed for both structure and texture color image retrieval based on FRAR model with Bayesian approach. The model coefficients are computed using the circular functions sine and cosine as discussed in section 2, the proposed scheme characterizes the structure and texture primitives in the periodic texture patterns also. Since the FRAR model characterizes the texture primitives and provides unique decimal number, it matches exact images in the image database and retrieves. Because, the proposed system facilitates the user to fix the level of significance for the test statistic $\mathrm{T}_{\mathrm{d}}^{2}$, the user can fix the significance level $\alpha$ at a desired level by which the user can retrieves only the required same or similar images, and not all the relevant images in the database as in the other existing systems. For example, at or below the significance level $0.001(1 \%)$, the system retrieves only the same image, and the rotated and scaled images of the same query image. But there is one disadvantage that if the same image is in the database, it retrieves the image; otherwise, it results that no image is matching with the query image. This problem can be overcome by fixing the significance level $\boldsymbol{\alpha}$ at more than 0.001 , by which the system retrieves the similar images. The user can fix the significance level himself at his convenient. Because the proposed system is a distributional approach, it is also invariant for rotation and scaling.

\section{REFERENCES}

[1] Qiu, G and Lam, K-M, (2003). "Frequency Layered Color Indexing for Content-Based Image Retrieval”, IEEE Transactions on Image Processing, Vol. 12, No. 1, pp. 102- 113.

[2] Xie, Y., Lu, H. and Yang, M-H., (2013) "Bayesian Saliency via Low and Mid-Level Cues", IEEE Transactions on Image Processing, Vol. 22, No 5, pp. 1689-1698.

[3] Zujovic, J., Pappas, T. N. and Neuhoff, D. L., (2013) "Structural Texture Similarity Metrics for Image Analysis and Retrieval", IEEE Transactions on Image Processing, Vol. 22, No. 7, pp. 2545-2558.

[4] Y. D. Chun, N. C. Kim, I. H. Jang, (2008), "Content-based image retrieval using multi-resolution color and texture features", IEEE Trabsaction on Image Processing, Vol. 10, No.6, pp. 1073-1084.

[5] Mustafa Ozden and Ediz Polat, (2007) "A color image segmentation approach for content-based image retrieval, "Pattern Recognition, Vol. 40, pp. 1318-1325.

[6] Yung-Kuan Chan, Yu-An Ho, Yi-Tung Liu and Rung-Ching Chen, (2008) "A ROI image retrieval method based on CVAAO," Image and Vision Computing. Vol. 26, pp. 1540-1549.

[7] B. M. Mehtre, M. S. Kankanhalli, W. F. Lee, (1997a), "Shape measures for content based image retrieval: A comparison,” Information processing and Management, Vol. 33, pp. 319-337.

[8] B. M. Mehtre, M. S. Kankanhalli, W. F. Lee, (1997b) "Content-based image retrieval using a composite color-shape approach, 'Information processing and Management, Vol. 34, pp. 109-120.

[9] X. Wan, C. C. J. Kuo, (1998), "New approach to image retrieval with hierarchial color clustering, "IEEE Transactions on Circuits and Systems for Video Technology, Vol. 8, pp. 628-643.

[10] P. Wu, B. S. Manjunath, S. Newsam, H. D. Shin, (2000) "A texture descriptor for browsing and similarity retrieval, "Signal Processing.,Vol. 16, pp. 33-43.

[11] N. Nikolaou, N. Papamarkos, (2002) "Color image retrieval using a fractal signature extraction technique, ”Engineering Applications of Artificial Intelligence, Vol. 15, pp. 81-96. 
[12] M. Kokare, B.N. Chatterji, P.K. Biswas, (2003) "Comparison of similarity metrics for texture image retrieval, " Proc. IEEE Transactions on TENCON 2003, Conference on Convergent Technologies for Asia-Pacific Region, Vol. 2, pp. 571-575.

[13] K. Seetharaman and N. Palanivel, (2013) "Texture characterization, representation, description and classification based on a family of full range gaussian markov random field model, "International Journal of Image and Data Fusion, Vol. 4, No. 4, pp. 1-24.

[14] T.W. Anderson, An Introduction to Multivariate Statistical Analysis, third ed., John Wiley \& Sons, Inc., 2003.

\section{AUTHORS}

A.Annamalai Giri pursued his MCA from University of Madras, Chennai, India in 2000 and M.Tech.(Information Technology) from Sathyabama University, Chennai India in 2007. Currently, am pursuing my Ph.D. research degree in M.S. Uuniversity, India, and working as Associate Professor of Computer Science at Sri Kuvempu First Grade College, Bangalore,India. His research interests include Statistical Model based Image Mining. Pattern Recognition, Digital Image Analysis, and Data Mining.

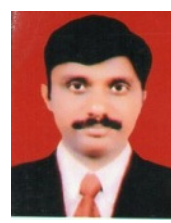

Dr.K.Seetharaman received his M.Sc. degree in Statistics from Annamalai University, hidambaram, India in 1990 and the M.S. degree in Software Systems from Birla Institute of Technology and Science, Pilani, India in 1995. He received his Ph.D. degree in Computer Science \& Engg. from Annamalai University in 2006.He is currently working as Associate Professor in the Department of Computer Science \& Engg., Annamalai University. His research interests include Statistical Pattern Recognition, Scene Analysis, Digital Image Analysis, and Data Mining and Knowledge Discovery. He is also actively involved in professional activities, viz. reviewer in journals: Pattern Recognition, Knowledge-based Systems, IEEE Transactions on Pattern Analysis and Machine Intelligence, $\mathrm{He}$ is currently serving as Associate Editor of the International Journal - Aloy Journal of Soft Computing and Applications, and the Editorial Board Member of the International Journal of Information Technology and Management Sciences. He is also the member of the professional societies - IEEE, ACM, IAENG, and Indian Science Congress Association. 